\title{
Rate of presence of 11 thoracic vertebrae and 6 lumbar vertebrae in asymptomatic Chinese adult volunteers
}

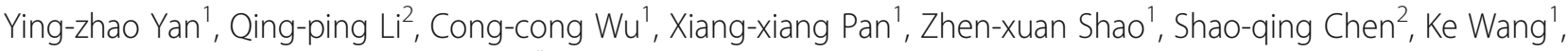 \\ Xi-bang Chen ${ }^{1}$ and Xiang-yang Wang ${ }^{1 *}$
}

\begin{abstract}
Background: An increasing number of studies on spinal morphology in asymptomatic Asian and Western patients have been reported. Variation in spinal anatomy among patients is considered as the cause of wrong-level surgery in up to $40 \%$ of cases. The present study examined the rate of presence of 11 thoracic vertebrae and 6 lumbar vertebrae in 293 asymptomatic Chinese adult volunteers.

Methods: From May 27, 2016, to November 11, 2017, a cohort of 325 asymptomatic Chinese adults meeting the study exclusion criteria was recruited. The radiographs were examined by a spine surgeon and a radiologist to assess the number of thoracic and lumbar vertebrae.

Results: In total, 293 volunteers were included in this study: 17 (5.8\%) had 11 thoracic vertebrae, and 16 (5.5\%) had 6 lumbar vertebrae. Among all volunteers, 12 (4.1\%) had 7 cervical vertebrae (C), 11 thoracic vertebrae (T), and 5 lumbar vertebrae (L); 5 (1.7\%) had 7C, 11T, and 6L; and 11 (3.8\%) had 7C, 12T, and 6L. There was no difference between the findings of the spine surgeon and the radiologist.

Conclusions: For the first time, this study describes the rate of presence of 11 thoracic vertebrae and 6 lumbar vertebrae in 293 asymptomatic Chinese adult volunteers. Variations in the number of thoracic and lumbar vertebrae tend to be ignored by spine surgeons. We encourage spinal surgeons and researchers to be aware of such variations when performing thoracic- and lumbar-level surgery and assessing spinal alignment and parameters.
\end{abstract}

Keywords: 11 thoracic vertebrae, 6 lumbar vertebrae, Wrong-site surgery, Spinal alignment, Asymptomatic volunteers

\section{Background}

Studies on the spinal morphology and alignment of asymptomatic Asians and Westerners are being reported with increasing frequency [1-9]. However, many such studies do not consider variations in the number of vertebrae, which can also lead to wrong-site surgery. It has been reported that as many as $50 \%$ of spinal surgeons have performed incorrect vertebral level surgery during their careers [10-12]. Variation in patient anatomy is considered the cause in up to $40 \%$ of cases of wrong-level surgery $[11,13]$.

\footnotetext{
* Correspondence: xiangyangwang@wmu.edu.cn

${ }^{1}$ Department of Orthopaedic Surgery, Zhejiang Spine Surgery Centre, The Second Affiliated Hospital and Yuying Children's Hospital of Wenzhou Medical University, 109 Xueyuanxi Rd, Wenzhou 325027, Zhejiang, China Full list of author information is available at the end of the article
}

Many surgeries are carried out in the spinal center of our hospital each year, primarily for fracture reduction, discectomy, and scoliosis correction. To avoid wrong-level surgery, we preoperatively check whole-spine images with cephalocaudal enumeration. The aims of this study were to describe the rate of presence of 11 thoracic vertebrae and 6 lumbar vertebrae in Chinese asymptomatic adult volunteers and to encourage spinal surgeons to be aware of the variations in the numbers of thoracic and lumbar vertebrae when performing thoracic- and lumbar-level localization and measuring spinal parameters.

\section{Methods \\ Subject enrollment and data collection}

This study received institutional review board approval and followed the principles of the Declaration of Helsinki. 
From May 27, 2016, to November 11, 2017, a cohort of 325 asymptomatic Chinese adults was recruited who were aged above 18 years and satisfied the following exclusion criteria: (1) lameness or unequal length of the lower limbs; (2) apparent scoliosis; (3) history of trauma of the spine, pelvis, or lower extremity; (4) history of hip or knee arthroplasty and spine, pelvis, or lower-limb surgery; (5) complaints of back pain, neck pain, or limb numbness caused by degenerative diseases of the spine, such as disc herniation, spinal canal stenosis, and lumbar spondylolisthesis; (6) strabismus or torticollis affecting balance; (7) history of neuromuscular disorders or congenital abnormalities; or (8) pregnancy or preparation for pregnancy.

Informed consent was obtained from each volunteer prior to the enrollment in this trial.

The volunteers were entitled to a free full-spine photograph and X-ray report, including of the chest, lungs, spine, and abdomen, in return for their participation.

\section{Radiographic analysis}

Full-spine standing anteroposterior and lateral radiographs were acquired for all volunteers with their arms in the fists-on-clavicles position. The radiographs were examined by a spine surgeon and a radiologist who had independently reviewed several hundred whole-spine images prior to this review.

In the posteroanterior view, the top rib was regarded as the first thoracic level, and enumeration proceeded caudally. Thoracic vertebrae were identified according to the corresponding rib attachments. All vertebrae with rib attachments, including the bilateral or unilateral ribs, were counted as thoracic vertebrae. A vertebra was considered to be at the lumbar vertebrae level only if it was not attached to the ribs.

Continuing caudally, the first lumbar vertebra was that following the last thoracic vertebra. When there was a complete vertebra between L5 and the sacrum, and well-formed disc material extending between the vertebra and sacrum, the vertebral body was defined as L6. Lumbosacral transitional vertebrae (LSTV) were defined based on previous literature [13, 14], i.e., one or both transverse processes attached to the sacrum through incomplete or complete osseous fusion or via a diarthrodial joint.

\section{Statistical analyses}

The SPSS statistical software package (ver. 19.0; SPSS Inc., Chicago, IL, USA) was used for the statistical analyses. Demographic data conforming to a normal distribution were expressed as means \pm standard deviation. Patient demographic characteristics including age, weight, height, and body mass index (BMI) were compared using independent samples $t$ tests. Variables expressed as frequencies were compared using the chi-squared test. $p$ values less than 0.05 were deemed to indicate statistical significance.

\section{Results}

Thirty-two volunteers had missing X-ray images or did not meet the exclusion criteria (Fig. 1). In total, 293 volunteers were included in this study.

The spine surgeon confirmed that two volunteers had only four lumbar vertebrae, whereas the radiologist believed there were no four-lumbar vertebrae volunteers. In addition, differences between the surgeon and radiologist were seen in the results of the vertebral body count, even though they both used the same method (see Table 1).

Differences were reviewed by the surgeon and radiologist, and a consensus was obtained in all cases. Twenty-eight (9.6\%) of the volunteers had an atypical number of thoracic and/or lumbar vertebrae. Seventeen (5.8\%) volunteers had 11 thoracic vertebrae, and 16 (5.5\%) had 6 lumbar vertebrae. An LSTV was present in nearly all patients who had an atypical number (i.e., six) of lumbar vertebrae (15 of 16 ; $93.8 \%)$. In total, 5 (1.7\%) of the 293 volunteers had an atypical number of both thoracic (11) and lumbar (6) vertebrae. No volunteer had 13 thoracic vertebrae and/or 4 lumbar vertebrae (Fig. 1).

Based on the above results, the volunteers were divided into four groups: group $7 \mathrm{C}+11 \mathrm{~T}+5 \mathrm{~L}$, group $7 \mathrm{C}+11 \mathrm{~T}+$ $6 \mathrm{~L}$, group $7 \mathrm{C}+12 \mathrm{~T}+5 \mathrm{~L}$, and group $7 \mathrm{C}+12 \mathrm{~T}+6 \mathrm{~L}$. The $\mathrm{X}$-ray images of each group are shown in Fig. 2.

The patient demographic data are included in Table 2. The volunteers had a mean age of $40.8 \pm$ 12.8 years (range 24 to 74 years), with a mean height of $164.0 \pm 7.0 \mathrm{~cm}$ (range 148 to $186 \mathrm{~cm}$ ), weight of $61.1 \pm 9.6 \mathrm{~kg}$ (range 40 to $95 \mathrm{~kg}$ ), and BMI of $22.6 \pm$ $2.8 \mathrm{~kg} / \mathrm{m}^{2}$ (range 16.2 to $32.9 \mathrm{~kg} / \mathrm{m}^{2}$ ). In total, 111 (38\%) volunteers were male, and 182 (62\%) were female. The differences in weight, height, and BMI between group $7 \mathrm{C}+11 \mathrm{~T}+5 \mathrm{~L}$, and group $7 \mathrm{C}+12 \mathrm{~T}+$ $5 \mathrm{~L}$ were statistically significant $(p<0.05)$.

\section{Discussion}

A previous study reported that approximately $10-17.4 \%$ of adults have some form of spinal abnormality, the most common of which is the presence of L6 $[1,5]$. Another large-scale study reported that people with LSTV account for 4-30\% of the general population [15]. In addition, approximately 5-8\% of "normal" individuals lack a pair of ribs/thoracic vertebrae [16], while additional ribs are sometimes considered as normal variants. However, we found no study of normal individuals having 11 thoracic vertebrae combined with 6 lumbar vertebrae. This study was a cross-sectional analysis of the rate of presence of 11 thoracic vertebrae and 6 lumbar vertebrae among 293 healthy subjects, as visualized 


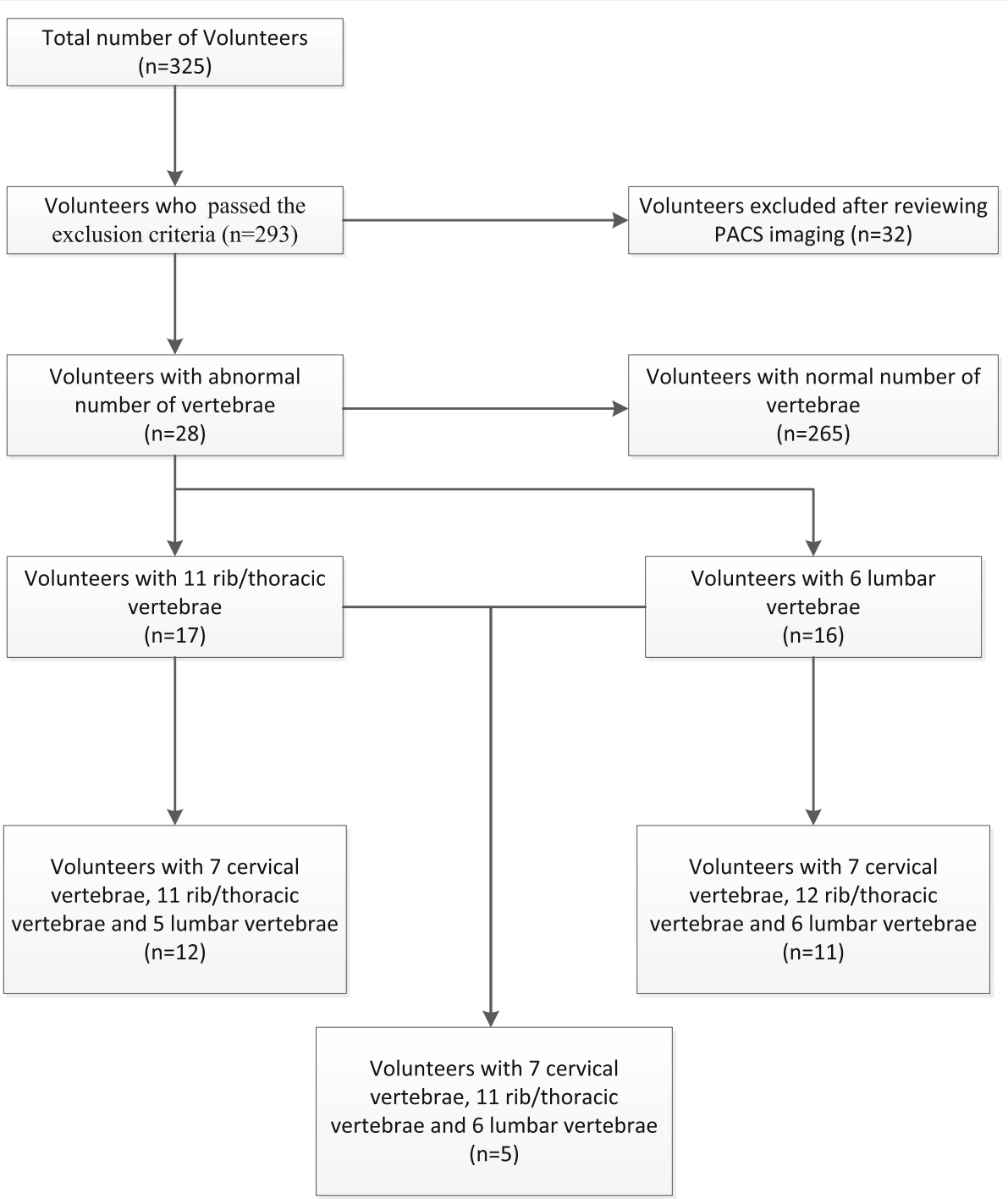

Fig. 1 A schematic illustrating the research process and distribution of asymptomatic volunteers with atypical numbers of vertebrae

Table 1 The results of a variable number of vertebrae by spine surgeon and radiologist

\begin{tabular}{llll}
\hline & Spine surgeon & Radiologist & $\begin{array}{l}\text { After joint } \\
\text { reconfirmation }\end{array}$ \\
\hline $7 C+11 T+5 \mathrm{~L}$ & $11(3.8 \%)$ & $12(4.1 \%)$ & $12(4.1 \%)$ \\
$7 \mathrm{C}+11 \mathrm{~T}+6 \mathrm{~L}$ & $12(4.1 \%)$ & $11(3.8 \%)$ & $5(1.7 \%)$ \\
$7 \mathrm{C}+12 \mathrm{~T}+4 \mathrm{~L}$ & $2(0.7 \%)$ & 0 & 0 \\
$7 \mathrm{C}+12 \mathrm{~T}+6 \mathrm{~L}$ & $12(4.1 \%)$ & $10(3.4 \%)$ & $11(3.8 \%)$ \\
$7 \mathrm{C}+12 \mathrm{~T}+5 \mathrm{~L}$ & $256(87.4 \%)$ & $260(88.7 \%)$ & $265(90.4 \%)$ \\
Total & 293 & 293 & 293 \\
\hline
\end{tabular}

There was no statistical difference between spine surgeon and radiologist $C$ cervical vertebrae, $T$ thoracic vertebrae, $L$ lumbar vertebrae on full-spine standing radiographs. We found that $9.6 \%$ of the asymptomatic population had an atypical number of thoracic and/or lumbar vertebrae. Among all volunteers, $4.1 \%$ were included in the $7 \mathrm{C}+11 \mathrm{~T}+5 \mathrm{~L}$ group, $1.7 \%$ in the $7 \mathrm{C}+11 \mathrm{~T}+6 \mathrm{~L}$ group, and $3.8 \%$ in the $7 \mathrm{C}+12 \mathrm{~T}+6 \mathrm{~L}$ group. Thus, $5.8 \%$ of the volunteers had 11 thoracic vertebrae, and 5.5\% had 6 lumbar vertebrae. We have to thank the reviewers of this article for helping us reconfirm the number of cases in group $7 \mathrm{C}+11 \mathrm{~T}+6 \mathrm{~L}$. Our findings are consistent with those of the previous studies $[15,16]$. However, the height and weight of the volunteers with 11 thoracic vertebrae and 5 lumbar vertebrae were significantly lower than those of the volunteers with a normal number of vertebrae. We believe that a reduction in the number of thoracic vertebrae has a great effect on body length and body size. 


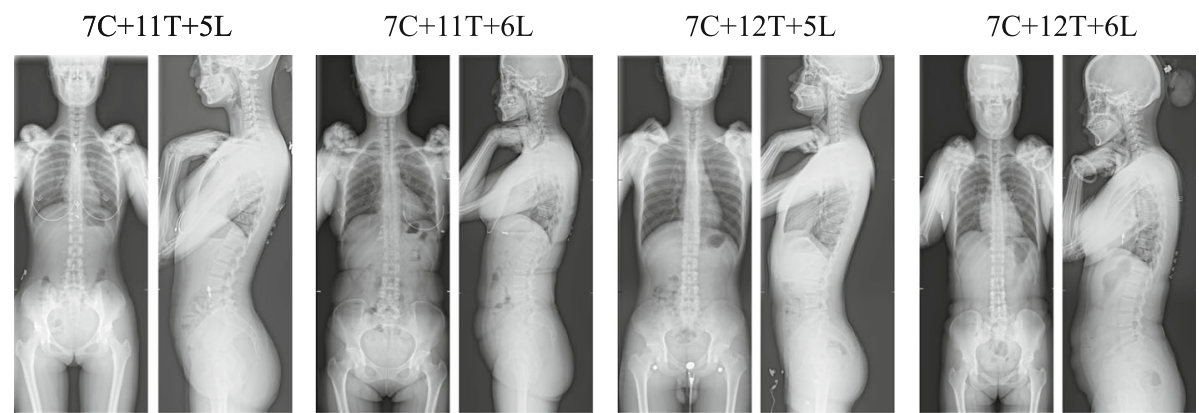

Fig. 2 Full-spine X-ray images of each group

Studies on the spinal morphology and alignment are being reported with increasing frequency [1-9]. More than 80 articles published in the past 5 years were retrieved from the PubMed database by a search including the keywords "asymptomatic," "spine," and "alignment." These studies measured spinal parameters, including occipitocervical alignment [2], cervicothoracic alignment $[2,7]$, spinopelvic alignment $[5,8]$, cervical parameters [6], thoracic parameters [6,9], and lumbar parameters [4], and described their role in spinal balance or the diagnosis and treatment of spinal diseases. However, although these studies used various measures to reduce errors when measuring spinal parameters, such as the establishment of exclusion criteria, and performance of multiple measurements by various experts, many of them ignored the important question of whether spinal parameters are measured accurately when there is variation in the number of vertebrae among patients [1-9]. For example, Mizutani et al. did not explain in their Methods section how they accounted for an absence of thoracic vertebrae, and therefore, we are unsure of how they dealt with that situation [9]. Yokoyama et al. [5] described the rate of presence of six lumbar vertebrae among a Japanese population and regarded the sacrum below the LSTV as a marker to evaluate the spinopelvic alignment of six lumbar vertebrae. Although an intervertebral disc exists between the L6 vertebra and the inferior sacral vertebra, mobility between the L6 vertebra and sacrum may be restricted [17]. When measuring the thoracic parameters of 11 thoracic vertebrae individuals, replacing T12 with T11 is the first intuition. However, if both 11 thoracic vertebrae and L6 are present, and L6 did not originate in S1, the superfluous first lumbar vertebra may be the last thoracic vertebra lacking ribs. In such cases, it seems appropriate to replace T12 with L1. Therefore, we suggest that, when collecting spinal alignment data from asymptomatic volunteers, exclusion criteria must be applied to exclude cases with an atypical number of vertebrae, even though these can account for $10-30 \%$ of all patients [5]. A spinal alignment database specifically pertaining to cases with an atypical number of vertebrae should be established. We remain skeptical of the article comparing global spinal alignment and balance between patients with atypical and normal numbers of vertebrae [5].

Wrong-level surgery is a sensitive and serious event for both the patients and the spine surgeons. Although over $50 \%$ of surgeons have performed wrong-level surgery during their career, many spinal surgeons still believe that it is completely avoidable [12]. Certain factors, including atypical anatomy, have been considered responsible for wrong-site spine surgery. Based on an analysis of 65 spinal surgery lawsuits, Goodkin et al. demonstrated that mistakes may arise due to omission

Table 2 Demographic characteristics of different groups

\begin{tabular}{|c|c|c|c|c|c|}
\hline & \multicolumn{4}{|l|}{ Group } & \multirow[t]{2}{*}{ Total } \\
\hline & $7 C+11 T+5 L$ & $7 \mathrm{C}+11 \mathrm{~T}+6 \mathrm{~L}$ & $7 C+12 T+6 L$ & $7 C+12 T+5 L$ & \\
\hline Number & 12 & 5 & 11 & 265 & 293 \\
\hline Male/female & $1 / 11$ & $1 / 4$ & $8 / 3$ & $101 / 164$ & $111 / 182$ \\
\hline Age & $35.9 \pm 14.2(23 \sim 70)$ & $42.8 \pm 9.9(29 \sim 53)$ & $40.2 \pm 13.9(24 \sim 63)$ & $40.9 \pm 12.8(20 \sim 74)$ & $40.8 \pm 12.8(20 \sim 74)$ \\
\hline Weight (kg) & $51.6 \pm 8.7^{* *}(41 \sim 67)$ & $58.7 \pm 2.7(55 \sim 62)$ & $60.5 \pm 8.9(49 \sim 75)$ & $61.5 \pm 9.6(40 \sim 95)$ & $61.1 \pm 9.6(40 \sim 95)$ \\
\hline Height (cm) & $160 \pm 6.0^{*}(155 \sim 175)$ & $163 \pm 3.8(158 \sim 167)$ & $167 \pm 8.0(154 \sim 180)$ & $164 \pm 7.0(148 \sim 186)$ & $164 \pm 7.0(148 \sim 186)$ \\
\hline BMI $\left(\mathrm{kg} / \mathrm{m}^{2}\right)$ & $19.9 \pm 2.2^{* *}(17.1 \sim 22.6)$ & $22.1 \pm 1.5(21.2 \sim 24.8)$ & $21.6 \pm 2.6(16.9 \sim 25.3)$ & $22.8 \pm 2.7(16.2 \sim 32.9)$ & $22.6 \pm 2.8(16.2 \sim 32.9)$ \\
\hline
\end{tabular}

The range is shown in parentheses; " $7 \mathrm{C}+12 \mathrm{~T}+5 \mathrm{~L}$ " stands for volunteers with normal number of vertebrae

$C$ cervical vertebrae, $T$ thoracic vertebrae, $L$ lumbar vertebrae

*Compared with group $7 \mathrm{C}+12 \mathrm{~T}+5 \mathrm{~L}, p<0.05$

${ }^{*}$ Compared with group $7 \mathrm{C}+12 \mathrm{~T}+5 \mathrm{~L}, p<0.01$ 
or misunderstanding of imaging studies performed before or during surgery [18].

An atypical number of vertebrae and the presence of LSTV may hamper accurate assessment of spinal anatomy. Approximately $5.5 \%$ of our asymptomatic volunteers showed an atypical L6 variation, in $93.8 \%$ of cases caused by LSTV. During the last 10 years, only one wrong-level surgery was conducted at our spinal surgery center among more than 5000 surgeries. In that patient, who was scheduled for L5/S1 segment surgery, we misperformed a decompression between L6 and the sacrum due to the presence of L6. The patient was not satisfied with the level of pain relief achieved after surgery; thus, as a remedial measure, selective nerve root block of L5/S1 was performed after communicating with the patient. Previous studies have also noted the role of LSTV in wrong-level discectomies. In some series, wrong-level discectomies due to variations in the number of vertebrae accounted for $40-71 \%$ of all procedures $[10,11]$. If 11 thoracic vertebrae are present, the ability to determine the surgical level before or during surgery based on cephalocaudal enumeration will be affected by changes in the thoracic vertebrae. Although researchers have proposed several lumbar localization methods for thoracolumbar surgery [12, 19], Longo suggests that further strategies are needed to reduce the risk of wrong-level surgery [20]. Mody et al. [10] made three recommendations to surgeons: direct communication with the patients before surgery, marking of predetermined sites, and use of verification radiographs.

In this study, there was a difference between the spine surgeon and radiologist in the ability to discriminate among vertebral variations, although the difference was not statistically significant. Considering the high $(9.6 \%)$ incidence of an atypical number of vertebrae, we recommend that spinal surgeons should not rely on the radiologist's report alone; ideally, the radiologist and surgeon should preoperatively determine the number of vertebrae together. More conveniently, the surgeon can associate the preoperative findings with the intraoperative $\mathrm{X}$-ray films.

To our knowledge, this is the first study of asymptomatic patients showing variation in the number of thoracic and lumbar vertebrae. However, some weaknesses of the study should be acknowledged. First, the sacrum is tilted at about $40^{\circ}$ in full-spine upright radiograph, so that it is difficult to evaluate L6 or LSTV. Regardless of how carefully we examined these radiographs, there could be a certain amount of misdiagnosis. Besides, we could not confirm that the vertebra variants were indeed LSTV by computed tomography (CT). So, we have to make a serious statement about the potential misdiagnosis of T12, L6, or LSTV in upright radiographs. It must be clear to all readers of this article that the data provided above is just a bit closer to the truth. Second, we cannot be sure that the incidence of variations in the number of thoracic or lumbar vertebrae in our limited sample is representative of the rate of such variations among the general population of eastern China; this remains to be confirmed by other researchers. Third, we did not include any patients with cervical ribs, 4 lumbar vertebrae, or 13 thoracic vertebrae. As expected, our selection criteria excluded these particular vertebrae variants. The rate of presence of cervical ribs varies from 0.05 to $8 \%$ in the general population, and they are rarely symptomatic in early childhood; however, in older children and adults, thoracic outlet syndrome or aneurysm formation can occur $[16,21,22]$. Supernumerary ribs, seen in trisomy 21 syndrome, are rarely seen as a normal variant [16]. Thus, in choosing adult asymptomatic volunteers, we excluded such variations.

\section{Conclusion}

For the first time, this study reported the rate of presence of 11 thoracic vertebrae and 6 lumbar vertebrae in 293 asymptomatic Chinese adult volunteers. We found that $4.1 \%$ of the patients were included in the $7 \mathrm{C}+11 \mathrm{~T}$ $+5 \mathrm{~L}$ group, $1.7 \%$ in the $7 \mathrm{C}+11 \mathrm{~T}+6 \mathrm{~L}$ group, and $3.8 \%$ in the $7 \mathrm{C}+12 \mathrm{~T}+6 \mathrm{~L}$ group. These variations tend to be ignored by spine surgeons; thus, we suggest that spinal surgeons and researchers should be aware of the variations in the number of thoracic and lumbar vertebrae when performing thoracic- and lumbar-level surgery and when assessing spinal alignment and parameters.

\section{Abbreviations}

C: Cervical vertebrae; L: Lumbar vertebrae; LSTV: Lumbosacral transitional vertebrae; T: Thoracic vertebrae

\section{Acknowledgements}

We would like to thank Ai-min Wu, MD, PhD, The Second Affiliated Hospital and Yuying Children's Hospital of Wenzhou Medical University, for his thoughtful discussions.

\section{Funding}

This work is supported by a major science and technology program for medical and health of Zhejiang Province (Grant No.WKJ-ZJ-1527).

\section{Authors' contributions}

YZY and XYW designed the study. YZY, QPL, XXP, ZXS, SQC, CCW, XBC, and $\mathrm{KW}$ recruited the volunteers. $Y Z Y$ and $Q P L$ examined the $X$-ray imaging. $X X P$ and ZXS performed the statistical analysis and interpreted the results. YZY drafted the manuscript. All of the authors critically revised the manuscript. The final manuscript was approved by all authors.

Ethics approval and consent to participate

This study was approved by the IRB (Institutional Review Board) of the Second Affiliated Hospital and Yuying Children's Hospital of Wenzhou Medical University (approval number: 2016-10), and the informed consent was signed by all volunteers.

Competing interests

The authors declare that they have no competing interests. 


\section{Publisher's Note}

Springer Nature remains neutral with regard to jurisdictional claims in published maps and institutional affiliations.

\section{Author details}

'Department of Orthopaedic Surgery, Zhejiang Spine Surgery Centre, The Second Affiliated Hospital and Yuying Children's Hospital of Wenzhou Medical University, 109 Xueyuanxi Rd, Wenzhou 325027, Zhejiang, China. ${ }^{2}$ Department of Radiology, The Second Affiliated Hospital and Yuying Children's Hospital of Wenzhou Medical University, 109 Xueyuanxi Rd, Wenzhou 325027, Zhejiang, China.

Received: 1 April 2018 Accepted: 15 May 2018

Published online: 23 May 2018

\section{References}

1. Dominguez D, Faundez A, Demezon H, Cogniet A, Huec JCL. Normative values for the $L 5$ incidence in a subgroup of transitional anomalies extracted from 147 asymptomatic subjects. Eur Spine J. 2016;25(11):1-6. https://doi.org/10.1007/s00586-015-4371-6.

2. Iyer S, Lenke LG, Nemani VM, et al. Variations in occipitocervical and cervicothoracic alignment parameters based on age: a prospective study of asymptomatic volunteers using full-body radiographs. Spine. 2016;41(23): 1837. https://doi.org/10.1097/BRS.0000000000001644.

3. Laouissat F, Sebaaly A, Gehrchen M, Roussouly P. Classification of normal sagittal spine alignment: refounding the Roussouly classification. Eur Spine J. 2017;1:1-10. https://doi.org/10.1007/s00586-017-5111-x.

4. Menezesreis R, Bonugli GP, Dalto VF, Herrero CF, Defino HL, Nogueirabarbosa $\mathrm{MH}$. The association between lumbar spine sagittal alignment and L4-L5 disC degeneration among asymptomatic young adults. Spine. 2016;41(18):E1081-7. https://doi.org/10.1097/BRS.0000000000001568.

5. Yokoyama K, Kawanishi M, Yamada M, et al. Spinopelvic alignment and sagittal balance of asymptomatic adults with 6 lumbar vertebrae. Eur Spine J. 2016;25(11):3583-8. https://doi.org/10.1007/s00586-015-4284-4.

6. Yu M, Zhao WK, Li M, et al. Analysis of cervical and global spine alignment under Roussouly sagittal classification in Chinese cervical spondylotic patients and asymptomatic subjects. Eur Spine J. 2015;24(6):1265-73. https://doi.org/10.1007/s00586-015-3832-2.

7. Lee SH, Kim KT, Seo EM, Suk KS, Kwack YH, Son ES. The influence of thoracic inlet alignment on the craniocervical sagittal balance in asymptomatic adults. J Spinal Disord Tech. 2012;25(2):E41. https://doi.org/10.1097/BSD. Ob013e3182396301.

8. Hwd H, Tan KA, Kantharajanna SB, et al. Using spinopelvic parameters to estimate residual lumbar lordosis assuming previous lumbosacral fusion-a study of normative values. Spine J. 2018;18(3):422-9. https://doi.org/10.1016/ j.spinee.2017.08.232.

9. Mizutani J, Verma K, Endo K, et al. Global spinal alignment in cervical kyphotic deformity: the importance of head position and thoracolumbar alignment in the compensatory mechanism. Neurosurgery. 2017; https://doi. org/10.1093/neuros/nyx288.

10. Mody MG, Nourbakhsh A, Stahl DL, Gibbs M, Alfawareh M, Garges KJ. The prevalence of wrong level surgery among spine surgeons. Spine. 2008;33(2): 194-8. https://doi.org/10.1097/BRS.0b013e31816043d1.

11. Jhawar BS, Mitsis D, Duggal N. Wrong-sided and wrong-level neurosurgery: a national survey. J Neurosurg Spine. 2007;7(5):467-72. https://doi.org/10. 3171/SPI-07/11/467.

12. Mayer JE, Dang RP, Duarte Prieto GF, Cho SK, Qureshi SA, Hecht AC. Analysis of the techniques for thoracic- and lumbar-level localization during posterior spine surgery and the occurrence of wrong-level surgery: results from a national survey. Spine Journal. 2014;14(5):741-8. https://doi.org/10. 1016/j.spinee.2013.06.068

13. Ibrahim DA, Myung KS, Skaggs DL. Ten percent of patients with adolescent idiopathic scoliosis have variations in the number of thoracic or lumbar vertebrae. Journal of Bone \& Joint Surgery-american Volume. 2013;95(9): 828-33. https://doi.org/10.2106/JBJS.L.00461

14. Plessis AMD, Greyling LM, Page BJ. Differentiation and classification of thoracolumbar transitional vertebrae. J Anat. 2018; https://doi.org/10.1111/ joa.12781.

15. Paik NC, Lim CS, Jang HS. Numeric and morphological verification of lumbosacral segments in 8280 consecutive patients. Spine. 2013:38(10):573-8. https://doi.org/10.1097/BRS.0b013e31828b7195.
16. Glass RB, Norton Kl, Mitre SA, Kang E. Pediatric ribs: a spectrum of abnormalities. Radiographics. 2002;22(1):87-104. https://doi.org/10.1148/ radiographics.22.1.g02ja1287.

17. Elster AD. Bertolotti's syndrome revisited. Transitional vertebrae of the lumbar spine. Spine. 1989;14(12):1373-7. https://doi.org/10.1097/00007632198912000-00015.

18. Goodkin R, Laska LL. Wrong disc space level surgery: medicolegal implications. Surg Neurol. 2004;61(4):323-41. https://doi.org/10.1016/j. surneu.2003.08.022.

19. Chin KR, Seale J, Cumming V. Avoidance of wrong-level thoracic spine surgery using sterile spinal needles. Clin Spine Surg. 2017;30(1):E54-8. https://doi.org/10.1097/BSD.0b013e3182a35762.

20. Longo UG, Loppini M, Romeo G, Maffulli N, Denaro V. Errors of level in spinal surgery: an evidence-based systematic review. Journal of Bone \& Joint Surgery British Volume. 2012;94(11):1546-50. https://doi.org/10.1302/ 0301-620X.94B11.29553.

21. Steiner HA. Roentgenologic manifestations and clinical symptoms of rib abnormalities. Radiology. 1943;40(2):175-8. https://doi.org/10.1148/40.2.175.

22. Brewin J, Hill $M$, Ellis $H$. The prevalence of cervical ribs in a London population. Clin Anat. 2009;22(3):331-6. https://doi.org/10.1002/ca.20774.

\section{Ready to submit your research? Choose BMC and benefit from:}

- fast, convenient online submission

- thorough peer review by experienced researchers in your field

- rapid publication on acceptance

- support for research data, including large and complex data types

- gold Open Access which fosters wider collaboration and increased citations

- maximum visibility for your research: over $100 \mathrm{M}$ website views per year

At BMC, research is always in progress.

Learn more biomedcentral.com/submissions 